BULLETIN OF THE

AMERICAN MATHEMATICAL SOCIETY

Volume 81, Number 2, March 1975

\title{
PROPER $T$-MAPS OF $T$-MODULES
}

\author{
BY ALAN A. MEYERHOFF
}

Communicated by Glen E. Bredon, October 24, 1974

In investigating homotopy equivalences of smooth $G$-manifolds where $G$ is a compact Lie group, Petrie [3], [4], [5] makes use of proper $G$-maps of degree 1 from one $G$-module to another of the same complex dimension. The first nontrivial example of such a map, called a quasi-equivalence, was given by Petrie [6] for two-dimensional $S^{\mathbf{1}}$-modules. Necessary and sufficient conditions for the existence of a quasi-equivalence when $G$ is any compact Lie group are now known [2]. For simplicity, the case where $G$ is a torus $T$ is outlined here.

Definitions and notation. Let $M$ and $N$ be $T$-modules of the same dimension, and let $\hat{T}$ be the group of irreducible $T$-modules. If there is a $T$-module $Q$ such that a quasi-equivalence $\omega: N+Q \rightarrow M+Q$ exists we say that there is a stable quasi-equivalence of $N$ to $M$, and we write $N \leqslant M$. Let $P=\left(p_{1}\right.$, $\cdots, p_{u}$ ) be a set of pairwise relatively prime integers with $u \geqslant 2$, or $P=(-1)$, and let $\mathbf{P}=\Pi\left(\psi^{p} i-1\right)$ be the associated Adams operation in $R(T)$, the complex representation ring of $T$. Petrie has conjectured this

THEOREM. $N \leqslant M$ iff there are nonnegative integers $a_{\mathrm{P}, \mathrm{\chi}}$ such that $M-N=\Sigma_{\chi \in \hat{T}} \Sigma_{\mathrm{P}} a_{\mathrm{P}, \chi} \mathbf{P} \chi$, in $R(T)$.

The proof [1] uses $K_{T}$-theory for the necessity in an argument suggested by Petrie. The sufficiency is shown by constructing the required maps.

Let any $T$-module $X$ be written as $X^{T}+X_{T}$, where $X^{T}$ is the set fixed pointwise by the action of $T$. If $N \leqslant M$, then the fact that $\omega: N+Q \rightarrow$ $M+Q$ is equivariant and proper leads, by an argument using a commutative diagram in $K_{T}$-theory, to

(i) $\operatorname{dim} M^{T}=\operatorname{dim} N^{T}$, and

(ii) $r(t)=\left(\lambda_{-1}\left(M_{T}\right)\right) /\left(\lambda_{-1}\left(N_{T}\right)\right) \in R(T)$, where $t=\left(t_{1}, \cdots, t_{n}\right) \in T$ represents the indeterminates in the expression for $R(T)$ as a Laurent polynomial ring over the integers.

AMS (MOS) subject classifications (1970). Primary 57D35, 57E25. 
The fact that $\omega$ has degree 1 requires

(iii) $|r(1)|=1$.

Now, each irreducible $T$-module in $M_{T}$ or $N_{T}$ contributes a factor of the form $\left(1-t^{x}\right)$ to $r(t)$, where $t^{x}=t_{1}^{x^{(1)}} \cdots t_{n}^{x^{(n)}}$ describes the action of $t \in T$, with the $x^{(i)}$ integers not all zero. These factors are partitioned into classes in the $j$ th of which all $x$ 's are multiples of a common $n$-vector $x_{j}$. Then the factors in that class are expressed as products of cyclotomic polynomials in the indeterminate $t^{x_{j}}$. Considerations of reducibility and the fact that $r(t)$ $\in R(T)$ require that all such cyclotomic polynomials in the denominator of $r(t)$ also appear in the numerator. After cancellation of such factors, what remains is a product of cyclotomic polynomials,

$$
r(t)=\prod_{j} \prod_{k} \phi_{m_{j, k}}\left(t^{x_{j}}\right) .
$$

The fact that deg $\omega=1$ requires that each $m_{j, k}$ not be a power of a prime. Then each $\phi_{m_{j, k}}$ can be written as a ratio of factors of the form $\left(1-\left(t^{x_{j}}\right)^{d}\right)$ with an equal number of factors in the numerator and denominator. Here the $d$ 's are positive integers determined by $m_{j, k}$. If we write $T$ modules

$$
M_{j, k}=\sum_{d \text { (numerator) }}\left(t^{x}\right)^{d} \text { and } N_{j, k}=\sum_{d \text { (denominator) }}\left(t^{x}\right)^{d},
$$

it is true that

$$
M_{j, k}-N_{j, k}=\prod_{h}\left(\psi^{p} h-1\right)\left(t^{x}\right)^{p}
$$

where $m_{j, k}=p \Pi_{h} p_{h}$ with the $p_{h}$ all the distinct prime factors. Then $\Sigma_{j, k}\left(M_{j, k}-N_{j, k}\right)$ is of the form given in the Theorem, and it is also equal to $M-N$.

For sufficiency, we observe that $M-N$ is expressed as the sum of terms of the form $\mathbf{P} \chi$ each of which can be thought of as some $M_{\mathrm{P}, \chi}-N_{\mathrm{P}, \chi}$. We construct $\omega_{\mathrm{P}, \mathrm{\chi}}: N_{\mathrm{P}, \mathrm{\chi}} \rightarrow M_{\mathrm{P}, \mathrm{\chi}}$ with the required properties, and take the direct sum of the maps, which is $\omega: N+Q \rightarrow M+Q$. For this construction, beginning with Petrie's two-dimensional example for $S^{1}$, which corresponds to $u=2$ in $P=\left(p_{1}, \cdots, p_{u}\right)$, we devise a $2^{u-1}$-dimensional quasi-equivalence and prove by induction on $u$ that it has the required properties [1]. The same map is good when $\chi \in \hat{T}$ too. The maps turn out to be polynomials in the complex variables and their conjugates, with normalizing adjustments and smoothing factors. Also, the above outline of a proof has assumed that in the 
irreducible $T$-modules $t^{x}$ each $x$ is a positive multiple of its $x_{j}$. The case where some are negative multiples can be treated by modifying the maps slightly.

The Theorem gives conditions for stable quasi-equivalences, but actual quasi-equivalences exist under virtually the same conditions [2].

\section{REFERENCES}

1. A. A. Meyerhoff, Proper equivariant maps of complex representation spaces of torus groups, Thesis, Rutgers University, May, 1974.

2. A. A. Meyerhoff and T. Petrie, Quasi-equivalence of G-modules (to appear).

3. T. Petrie, Equivariant quasi-equivalence, transversality and normal cobordism, Proc. Internat. Congress Math., Vancouver, 1974 (to appear).

4. Obstructions to transversality for compact Lie groups, Bull. Amer. Math. Soc. 80 (1974), 1133-1136.

5. actions on $P\left(C^{4 n}\right)$, Topology (to appear).

6. - Smooth $S^{1}$ actions on homotopy complex projective spaces and related topics, Bull. Amer. Math. Soc. 78 (1972), 105-153.

DEPARTMENT OF MATHEMATICS, RUTGERS UNIVERSITY, NEW BRUNSWICK, NEW JERSEY 08903 